\title{
Etude des pratiques dans la prise en charge de la maladie de La Peyronie en France
}

\author{
B. Cuzin, E. AmaR*, M. Schouman**, J.M Dubernard \\ Service d'Urologie et de la Transplantation, Hôpital E Herriot, Place d'Arsonval, 69437 Lyon cedex \\ *17, Avenue Victor Hugo, 75116 Paris \\ ** Centre Urologique, 164, Avenue Charles de gaulle 92200 Neuilly
}

\section{RESUME}

Le comité d'andrologie de l'Association Française d'Urologie a réalisé une enquête afin d'évaluer la prise en charge de la maladie de La Peyronie en milieu urologique en 1996.

Une enquête prospective a été réalisée entre avril 1995 et septembre 1996 à l'aide d'un questionnaire, incluant tous les nouveaux patients vus en consultation pour ce motif.

201 patients ont été inclus, de moyenne d'âge de 55,3 ans (écart type: 12,96). Un diabète sucré était présent chez $15,75 \%$ des patients et une maladie de Dupuytren chez $12,5 \%$. La localisation des plaques, leur nombre et leur taille ont été décrits de même que la localisation de la coudure et les symptômes associés à la maladie.

Le premier symptôme de découverte de la maladie était la coudure isolée (35\%), suivi de l'impuissance isolée et de la coudure associée à la douleur $(17,5 \%$ chacun). Des investigations complémentaires ont été réalisées chez $37 \%$ des patients (principalement échographie des corps caverneux, pharmacotest, radio simple, imagerie par résonance magnétique). Les traitements les plus fréquemment prescrits en première intention ont été la vitamine $E(58 \%)$, les injections locales de corticoïdes $(18 \%) ; 15,5 \%$ des patients n'ont pas eu de traitement (abstention surveillance). Le suivi moyen a été de
6,8 mois (écart type: 5,09 ). Globalement: $24 \%$ des patients ont été perdus de vue, $12,5 \%$ ne sont pas évaluables (n'ayant eu qu'une seule visite), 30,5\% des patients sont améliorés, $23 \%$ stabilisés et $10 \%$ sont aggravés. Les différentes sous populations évolutives ont également été analysées en fonction de leur symptômes et leur traitement.

Mots clefs: Maladie de La Peyronie, étude des pratiques

Une étude des pratiques a été réalisée dans le cadre du comité d'andrologie de l'Association Française d'Urologie afin d'avoir une image actuelle de la prise en charge la maladie de La Peyronie, ainsi qu'une description des caractéristiques épidémiologiques de la maladie vue en milieu urologique.

\section{MATÉRIEL ET MÉTHODES}

Une étude de type suivi de cohorte a été réalisée de manière prospective. Tous les patients vus en première consultation pour maladie de La Peyronie entre le 1er avril 1995 et le 1er septembre 1996 par les centres participants ont été inclus dans l'étude.

Un fiche de recueil a été élaborée comportant les caractéristiques cliniques du patient (âge, antécédents pertinents, ainsi que les caracté- 
ristiques physiques de la maladie (durée d'évolution, localisation de la coudure, de la plaque, nombre de plaques; paramètres de biomécanique pénienne: L1 (longeur initiale), L2 (longeur post-étirement), Lmax (longeur d'étirement maximale) et extensibilité, circonférence en flacidité; caractéristiques cliniques (mode de découverte, symptômes): chaque symptôme était décrit sur une échelle d'intensité à plusieurs niveaux . L'érection a été classée en 5 grades (érection normale $=4$; pénis assez rigide pour pénétration mais pas totalement rigide $=3$; pénis pas assez rigide pour pénétration $=2$; tumescence mais pénis non rigide $=1$; pas d'érection du tout $=0$ ), la douleur au moment de l'érection a été également classée en 5 grades (pas du tout douloureuse; un peu; modérément; beaucoup; extrêmement), enfin l'existence d'une gêne mécanique a été recherchée.

Une partie suivi décrivait l'évolution des symptômes en 3 stades : aggravé, stabilisé, amélioré. Les formes ont également été classées en invalidantes et non invalidantes : la forme invalidante étant définie comme comportant au moins un des 3 symptômes suivants : une douleur de grade supérieure à modérément, érection de grade inférieure à 3 , déformation avec gêne mécanique.

L'amélioration clinique a été définie sur le dernier changement d'état rapporté, changement vers un état moins péjoratif ( passage d'aggravé à stabilisé ou de stabilisé à amélioré ou d'aggravé à amélioré) pour au moins un des symptômes.

La stabilisation clinique a été définie sur la dernier état clinique rapporté : état stable de tous les symptômes. L'aggravation clinique a été définie sur le dernier changement d'état rapporté, changement vers un état plus péjoratif (passage de stabilisé à aggravé à ou de stabilisé à amélioré ou d'amélioré à aggravé) pour au moins un des symptômes.

\section{RÉSULTATS}

201 patients ont eu un recueil de données correspondant aux périodes d'inclusion. En raison des données manquantes, les chiffres sont donnés en pourcentage. Les données man- quantes ne constituaient toutefois pas plus de $10 \%$ de l'effectif sauf pour les données concernant les paramètres de biomécanique de la verge.

\section{Données épidémiologiques descrip- tives sur les patients et la maladie:}

- Caractéristiques des patients :

L'âge moyen des patients était de 55,3 ans (écart type : 12,96); dans les antécédents médicaux il existait $15,75 \%$ de patients diabétiques, $12,5 \%$ de patients atteints de maladie de Dupuytren, $4,3 \%$ atteints d'hypertension artérielle, $1 \%$ des patients avaient eu des autoinjections intracaverneuses.

La durée de la maladie avant la première consultation avait été de 16 mois (écart type : 31,21 mois).

- Localisation de la coudure:

- dorsale : $55,5 \%$

- ventrale : $14 \%$

- gauche : $15,5 \%$

- avec deux composantes : $11 \%$

- droite : $4 \%$

- Localisation de la plaque:

- dorsale distale : $46 \%$

- dorsale proximale : $20 \%$

- 2 localisations : $15 \%$

- ventrale distale : $6 \%$

- ventrale proximale : $7,5 \%$

- septale : $5,5 \%$

- Autres caractéristiques physiques de la maladie:

- nombre de plaques : 1,3 (écart type : 19,5)

- L1 (mesurée chez 72 patients) : $101 \mathrm{~mm}$ ( écartype : 18,5 )

- L2 (mesurée chez 62 patients) : $118 \mathrm{~mm}$ (écart type : 20,6)

- L Max (mesurés chez 18 patients) : 132,3 mm (écart type : 35,3 )

- Extensibilité (mesurée chez 18 patients) : 
$39.5 \mathrm{~mm}$ (écart type : 34,7 )

- Circonférence (28 patients): $63,4 \mathrm{~mm}$ (écart type: 35,3 )

- Mode de découverte de la maladie : le mode le plus fréquent de découverte de la maladie a été :la déformation de la verge isolée : $35 \%$ des cas, suivie de l' impuissance isolée : $17,5 \%$ et de l' érection douloureuse associée à une déformation : $17,5 \%$, enfin de la déformation associée à une impuissance : $13 \%$.

Les autres formes ont été plus rares : érection douloureuse $(8,5 \%)$, association douleur, déformation, impuissance (5\%), découverte systématique $(2 \%)$, perception par le patient d'un nodule $(1 \%)$, érection douloureuse et impuissance $(0,5 \%)$.

\section{- Intensité des symptômes :}

Lorsque l'érection était douloureuse : elle était peu douloureuse dans $41,5 \%$ des cas, modérément douloureuse dans $21,5 \%$ des cas, très douloureuse dans $34 \%$ des cas et extrêmement douloureuse dans $3 \%$ des cas.

Lorsque la déformation était présente elle était responsable d'une gêne mécanique dans $66 \%$ des cas.

Lorsqu'il existait une insuffisance érectile, l'érection était de grade 0 dans $11 \%$ des cas, de grade 1 dans $22 \%$ des cas, de grade 2 dans $23 \%$ des cas, de grade 3 dans $44 \%$ des cas.

\section{Bilan réalisé}

- Des examens paracliniques ont été demandés chez $37 \%$ des patients : on a observé une grande variation en fonction des centres allant de centres ne demandant jamais aucun examen à un centre demandant des explorations à tous les patients. Il n'existait pas ,non plus, de protocole consensuel pour le type d'examen à demander, mais une spécificité selon le centre.

- L'échographie simple des corps caverneux a été l'examen le plus demandé ( $34 \%$ des examens paracliniques), suivie par le pharmacotest $(20,5 \%)$, les radios simples des corps caverneux $(16,5 \%)$ et le l'écho-doppler pénien $(13 \%)$. Les autres examens étaient plus rares : imagerie par résonance magné- tique $(11 \%)$, cavernométrie $(2.5 \%)$, et rigidimétrie nocturne informatisée $(2.5 \%)$.

\section{Prises en charge thérapeutique}

- La première attitude thérapeutique proposée a été principalement le traitement médicamentaux peros : $51 \%$ des cas, puis les injections locales dans la plaque : $18 \%$ des cas, suivi de l'abstention surveillance $(15,5 \%)$. D'autres traitements ont également été réalisés : chirurgie (9\%), association traitement per os et injections locales (4\%), pompe à vide ( 2 cas), injections intracaverneuse (1 cas), ionophorèse (1 cas), association traitement per os et chirurgie (1 cas), association per os et radiothérapie localisée (1 cas).

- descriptif des traitements utilisés:

- le traitement per os utilisait la prescription de vitamine $\mathrm{E}$ essentiellement (environ la moitié des prescriptions per os), une association de vitamine $\mathrm{E}$ et de saponifiables de l'avocat (dans le quart des cas) et des saponifiables de l'avocat seul, du potaba (potassium para amino para benzoic acid), et divers dans le restant des cas.

- les injections locales utilisaient principalement les injections de corticoïdes à raison d'une par semaine ou d'une par mois.

- les types d'intervention chirurgicale réalisés ont été le Nesbit principalement mais également l'exérèse de la plaque suivie de greffe, la pose d'implants souples et autres prothèses péniennes.

- traitements proposés en fonction des symptômes :

- dans les formes douloureuses : le traitement per os a été principalement proposé $(58 \%$ des cas), puis les injections locales (24\% des cas). Les autres traitements ont été : abstention surveillance $(6 \%)$, chirurgie $(2 \%)$, association injections locales et radiothérapie $(2 \%)$, association injections locales et traitement per os $(8 \%)$

- dans les formes avec déformation : le traitement per os a été le plus prescrit $(46 \%)$, puis l'abstention surveillance (22\%), suivi des injections locales $(19 \%)$. Les autres traite- 
ments ont été la chirurgie $(6,5 \%)$, l'association injections locales et radiothérapie $(1,5 \%)$, l'association injection locale et per os $(5 \%)$

- dans les formes avec insuffisance érectile : le traitement le plus prescrit a été le traitement per os (55\%) suivi de l'abstension surveillance $(13 \%)$, des injections locales $(13 \%)$ et de la chirurgie $(12 \%)$. Les autres traitements ont été : vacuum $(3 \%)$ et injections intracaverneuses (4\%).

\section{Suivi, évolution}

Les données de suivi ont permis avec un recul moyen par rapport à la prise en charge de 6,8 mois (écart type : 5 mois) d'observer que $24 \%$ (48) des patients ont été perdus de vue, $30,5 \%(61)$ des patients ont été améliorés, $23 \%$ (47) stabilisés et 10\% (20) aggravés. Enfin $12,5 \%(25)$ des patients sont non évaluables (recul trop court)

Si l'on analyse l'évolution clinique pour chaque catégorie de patients en fonction de la gravité de la forme clinique de départ (forme invalidante / forme non invalidante), les éléments suivant sont observés :

- pour les patients améliorés (61 patients) : ces patients avaient dans $63 \%$ des cas au départ une forme invalidante. Le traitement en cours lors de l'évaluation finale était le traitement per os dans $39 \%$ des cas, puis les injections locales et la chirurgie dans $22 \%$ des cas chacun. Les autres traitements étaient : abstention surveillance $(4,5 \%)$, injections intracaverneuses $(10,5 \%)$, ionisation (2\%). Plus de $75 \%$ des patients n'ont eu qu'une 1 ou 2 visites de suivi, et un quart de 3 à 4 visites

- pour les patients stabilisés (47 patients) : ces patients avaient dans $71,5 \%$ des cas au départ une forme invalidante. Le traitement prescrit lors de l'évaluation finale était le traitement per os dans $40 \%$ des cas, des injections locales dans $34,5 \%$ des cas. Les autres traitements étaient la chirurgie $(9 \%)$, l'abstention surveillance $(3 \%)$, la ionophorèse $(2,5 \%)$, les injections intracaverneuses $(5,5 \%$ et la radiothérapie $(5,5 \%)$ des cas. Environ $80 \%$ des patients n'ont eu qu'une à deux visites de suivi et le restant de 3 à 4 visites.

Pour les patients perdus de vue (48 patients) : ces patients avaient des symptômes invalidants dans $62 \%$ des cas. Le traitement prescrit était l'abstention surveillance $(43 \%)$, le traitement per os $(27 \%)$. Les autres traitements étaient : les injections locales $(8 \%)$, la chirurgie $(8 \%)$, les injections intracaverneuses ( $8 \%$ ) et le vacuum $(6 \%)$. Environ $90 \%$ des patients ont eu de 1 à 2 visites et le restant de 3 à 4 visites

- Pour les patients aggravés (20 patients) : ces patients avaient des symptômes invalidants dans $100 \%$ des cas. Le dernier traitement prescrit était la chirurgie dans 6 cas, le traitement per os dans 5 cas, l'abstention surveillance dans 3 cas, les injections locales dans 4 cas, les injections intracaverneuses dans 2 cas.

\section{DISCUSSION}

L'âge moyen des patients de notre série est concordant avec les chiffres retrouvés dans la littérature $[1,2]$. De même le délai entre le début de la maladie et la première consultation est celui décrit dans la littérature: entre 6 et 24 mois, parfois il peut atteindre plusieurs années $[1,3]$. Les maladies décrites comme étant associées à la maladie de La Peyronie sont également retrouvées dans notre série: maladie de Dupuytren, diabète, hypertension artérielle $[2,4,5]$. L'association avec les arthrites $[2,6]$ n'est cependant pas retrouvée, il peut cependant s'agir d'un biais lors de l'interrogatoire, les arthrites n'étant pas forcément recherchées et signalées par les patients aussi facilement que les autres pathologies.

En ce qui concerne la clinique, la coudure de la verge est le symptôme le plus fréquent comme dans les autres séries de la littérature. La localisation dorsale la plus fréquente est elle aussi une donnée classique pour la coudure et la plaque [6].

En ce qui concerne les variations de longeur du pénis flaccide provoquées par étirement manuel maximum, si les valeurs des para- 
mètres L1 et L2 semblent proches de celles de la population normale [7], les valeurs des paramètres Lmax, extensibilité et circonférence, sont diminués traduisant le rôle de la fibrose sur la biomécanique pénienne et sur la structure de l'albuginée [8].

Le traitement par voie orale à base de Vitamine $\mathrm{E}$ est très utilisé dans la plupart des pays même si son efficacité est contestée. De même la stratégie abstention thérapeutique est pratiquée volontiers [2] dans cette pathologie dont le potentiel évolutif est imprévisible et le mécanisme physiopathologique très peu connu.

Le suivi des patients n'est pas suffisamment long pour tirer des informations fiables concernant le pronostic de la maladie: à court terme le pronostic semble plutôt favorable ( plus de 50\% des patients sont stabilisés ou améliorés) cependant presqu'un quart des patients sont perdus de vue. Dans la littérature, une évolution spontanée favorable a été observée dans $50 \%$ des patients environ chez des patients suivis pendant plusieurs années, cependant il s'agit de petites séries de patients $[6,9]$.

Il est impossible de tirer des conclusions concernant l'effet des traitements d'une étude telle que nous l'avons réalisée, d'autre part le suivi a été court. Dans l'ensemble en dehors de la déformation qui était le plus souvent responsable de gêne mécanique les autres symptômes étaient moins fréquents (environ $30 \%$ de patients présentaient des érections douloureuses ou une impuissance) et d'intensité moindre ( $44 \%$ d'érections de grade 3 et $63 \%$ de douleurs faibles ou modérées). Ces données associées au suivi court et à l'absence de traitement étiologique expliquent sans doute que les traitements symptômatiques ont été principalement les traitements par voie orale et l'abstention thérapeutique quel que soit le symptôme présenté par le patient. D'autre part, avant de proposer une correction chirurgicale pour une coudure de verge responsable de gêne mécanique il est admis qu'il faut attendre une période de stabilisation clinique de la coudure d'un an.

Enfin, les patients perdus de vue (presque le quart des patients porteurs de symptômes responsables de gêne identique aux autres groupes), l'ont été principalement après la première visite et étaient constitués d'une majorité de patients pour lesquels aucun traitement n'avait été prescrit. Même si là encore ce type d'étude ne permet de faire que des suppositions, on peut se demander si ce type de prise en charge n'a pas négligé l'aspect psychologique du retentissement de la maladie rapporté chez $77 \%$ des patients dans la littérature [10].

Notre étude a confirmé certaines données d'épidémiologie descriptive disponibles dans d'autres pays. Le suivi est trop court pour pouvoir décrire de manière fiable l'histoire de la maladie et particulièrement l'histoire naturelle de la maladie, cependant le pronostic plutôt favorable de la maladie à moyen terme semble vérifié. Ce type d'étude ne permet pas d'obtenir des données d'épidémiologie analytique qui permettraient de progresser dans la compréhension étiopathogénique de la maladie. La rareté de la maladie rend de plus, les études muticentriques indispensables.

Le nombre des patients perdus de vue pourrait illustrer la difficulté actuelle de la gestion médicale $d$ ' une maladie au vécu difficile, pour laquelle le traitement étiologique est inexistant et le traitement symptomatique pas toujours satisfaisant.

Remerciements aux membre du comité d'andrologie ayant participé à l'enquête : M Belicar (Amiens), P Bondil (Chambéry), D Chevalier (Nice), F Cour (Paris), D Delavierre (Orléans), M Etcheverry (Bordeaux), F Giuliano (Paris), J Hermabessière (Clermont Ferrand), P Menut, A Leriche (Lyon), JM Rigot (Lille), D Rossi (Marseille), L Sibert (Rouen), L Wagner, P Costa, H Navratil (Nimes, Montpellier)

\section{REFERENCES}

1. CP CHILTON, WM CASTLE, CA WESTWOOD, JP PRYOR Factors associated in the aetiology of Peyronie's disease Brit J Urol 1982, 54: 748.

2. MB LINDSAY, DM SCHAIN, P GRAMBSCH, RC BENSON, CM BEARD, LT KURLAND The incidence of Peyronie's disease in rochester, Minnesota, 1950 through 1984. J Urol 1991, vol 146: 1007.

3. EB EINER, JN KABALIN, R KESSLER Penile implants in the treatment of Peyronie's disease $J$ Urol 
$1991 ; 145: 69-72$

4. J CHESNEY Peyronie's disease Br J Urol 1975, 47: 209-218.

5. D DELAVIERRE, H IBRAHIM, M PENEAU La maladie de La Peyronie Encycl Med Chir (Elsevier, Paris), Néphrologie, urologie, 18-355-A-10. 1997, 6 p.

6. WL FURLOW, HE SWENSON, RE LEE Peyronie's disease: a study of its natural treatment and treatment with orthovoltage radiotherapy J Urol 1975, 114: 69-71.

7. P BONDIL, P COSTA, JP DAURES, JF LOUIS, NAVRATIL H Clinical study of the longitudinal deformation of the flaccid penis and of its variations with aging. Eur. Urol. 1992: 284-289.

8. G BROCK, GL HSU, L NUNES, B VON HEYDEN, TF LUE The anatomy of the tunica albuginea in the normal penis and Peyronie's disease J Urol 1997, 157: 276-281.

9. JL WILLIAMS, GG THOMAS The natural history of Peyronie's disease J Urol 1970, 103: 75-76.

10. MK GELBARD, F DOREY, $K$ JAMES The natural history of Peyronie's disease J Urol 1990, 144: 1376.

\section{ABSTRACT}

Survey on practice trends in the diagnosis and treatment of Peyronie's disease in France

B CuzIN, E AMAR*, M Schouman **, J M DUbERnARD

Objectives: the Andrology sub-commission of French Urological Association (AFU) has conducted a survey to describe urologist's practice in Peyronie's disease (PD).

Material and Methods: a prospective study has been done between April 1995 and September 1996 to evaluate the management of new patients seen during this period. After a description of clinical characteristics of the study group, initial treatments have been described .Outcomes according to patients characteristics have also been assessed using a functional classification (degree of functional discomfort).

Résults: 201 patients have been included: mean age 55.3 years (SD: 12,96). Diabetes mellitus was present in $15.75 \%$ and Dupuytren's contracture in $12.5 \%$. Plaque localization, number and size have been described just as the localization of curvature and symptoms. The first symptom at presentation was isolated penis curvature (35\%) followed by isolated impotence $(17.5 \%)$ then associated painful erection and penis curvature $(17.5 \%)$. Test were perform in $37 \%$ of the patients (duplex doppler ultrasonography, intracavernous injection, penile $X$ Rays, MRI). Main initial treatment prescribed was Vitamin $\mathbf{E}(58 \%)$, followed by intralesional injections (18\%); watchful waiting has been advocated in $15.5 \%$ of patients. Mean follow up has been 6.8 months (SD: 5.09). Globally, 24\% (48) of the patients have been lost to follow up, 12.5\% (25) have been not evaluable (only one visit), $30.5 \%(61)$ have been improved, $23 \%$ (47) stabilized and $10 \%$ (20) worsened. According to the functional classification used, gravity of disease were similar in improved, stabilized and lost to follow up subgroups but patients lost to follow up had mainly no treatment (watchful waiting).

Conclusions: this study has permitted to obtain some aspects of PD descriptive epidemiology in France, analytical epidemiology is not avalaible because of design of the study.Patients ought to be better informed about MD. At least, further follow up is needed to evaluate the history of disease.

Key-words: Peyronie's disease, practice trends, descriptive epidemiology 